\title{
La preservación digital sonora
}

\author{
Perla Olivia Rodríguez Reséndiz *
}

Artículo recibido:

7 de abril de 2014.

Artículo aceptado:

9 de abril de 2014.

\section{Resumen}

La preservación de los archivos sonoros está en un punto crítico de su historia debido a que día a día desaparecen miles de documentos sonoros como consecuencia del deterioro y la fragilidad de los soportes en que han sido grabados, así como por la obsolescencia de los equipos de grabación y reproducción analógica. Hasta ahora, la transferencia de contenidos analógicos a plataformas digitales (digitalización) es la única forma de garantizar la existencia del patrimonio sonoro; por ello la preservación digital de archivos sonoros constituye una forma de salvaguarda a largo plazo que ha modificado los métodos de preservación de documentos sonoros en soportes analógicos.

* InstitutodelnvestigacionesBibliotecológicasydelalnformacióndelaUNAM, México.perla@iibi.unam.mx

INVESTIGACIÓN BIBLIOTECOLÓGICA,Vol. 30,Núm.68,enero/abril,2016,México,ISSN: 0187-358X. pp. 173-195 
Palabras clave: Archivos sonoros; Preservación; Preservación digital; Preservación digital sonora; Digitalización; Documentos sonoros.

\begin{abstract}
Digital preservation of sound recordings

Perla Olivia Rodríguez-Reséndiz
\end{abstract}

Because thousands of sounds documents are lost every day as a result of the fragility and deterioration of recording supports, and the obsolescence of analog recording and playback equipment, the preservation of the sound archives is at a critical point in its history. Currently, the transfer of analog content to digital platforms is the only way to guarantee the survival of sound heritage. Therefore, the preservation of digital audio files constitutes a long-term safeguard that will eventually replace analog backup methods in order to preserve the world's audio heritage.

Keywords: Sound Archives; Preservation; Digital Preservation; Digital Sound Preservation; Digitizing; Sound Documents.

\title{
Introducción
}

D urante muchos años se consideró a los documentos sonoros, también denominadosfonorregistros, como documentos especiales frenteal dominio del documento textual; fueron definidos a partir del universo librario y conocidos como documentos no librarios. Esta situación se comprende si tomamos en consideración que los documentos sonoros son recién nacidos frente al libro. Las colecciones sonoras son jóvenes, comparadas con el patrimonio que se resguarda en los museos y en las bibliotecas, ya que comenzaron a crearse en el siglo XX (Wright, 2012).

Para Jean Weihs (2001) los documentos sonoros comenzaron a hacerse visibles a partir de los años 50 y 60 del siglo pasado, con particular incidencia en bibliotecas universitarias de Estados Unidos y Canadá. Derivado de la aparición de documentos sonoros, se crearon catálogos especiales en las bibliotecas. Dayli (1967) evidenció que los fonorregistros requerían nuevas 
formas de procesamiento, almacenamiento y catalogación, por ello desarrolló una crítica a las Reglas de Catalogación Angloamericana, editadas en 1967. Opinó que éstas eran insuficientes para la catalogación de documentos sonoros. Este primer análisis de Dayli, presentado a fines de los años 60, sentó las bases para considerar que las grabaciones sonoras eran documentos diferentes a los librarios y necesitaban un tratamiento especial por ser nuevos medios de información.

Rousseau y Couture (1994) sitúan a la década de 1960 a 1970 como el periodo en que los archivistas se interesaron verdaderamente en los documentos no textuales de sus archivos. De acuerdo con Ávila (2013), en la década de los años 70 el archivo vivió una extensión de sus dominios (por ejemplo, archivos administrativos, de negocios y privados) y la aparición de nuevos campos (los archivos sonoros, de imágenes y microfilms).

El desarrollo de la archivología y el interés por los documentos sonoros coincidió con la creación de la Asociación Internacional de Archivos Sonoros y Audiovisuales (IASA) en Ámsterdam en 1969. El surgimiento de la IASA tuvo como propósito contar con un foro para alentar la cooperación entre archivistas y bibliotecarios quienes tenían bajo su resguardo documentos sonoros.

La notoriedad de los archivos sonoros en el ámbito documental coincide con la incorporación progresiva y constante de las tecnologías de información y comunicación que Castells (2004) denomina la revolución del conocimiento, caracterizada porque la información es la base de la economía informacional. Este proceso se contextualiza en lo que Vivas Moreno (2013) denomina archivística integral en la sociedad dela información, entendido como el periodo en el que la disciplina experimenta un importante desarrollo en los principios conceptuales y en su aplicación, los cuales pueden verse plasmados en la ampliación del campo de actuación y la expansión científica de la archivística, en la consideración de que el archivo, la biblioteca o centro de documentación quedan englobados en el proceso informativo documental de las ciencias de la información y documentación y en la incorporación de las tecnologías de la información y la comunicación como cuestión intrínseca a las tareas archivísticas. Desde la perspectiva de Vieira (2013) la archivología posmoderna surgió a finales del siglo XX y se caracterizó por el desenvolvimiento y difusión de las tecnologías de información y comunicación, lo que condujo a los archivistas a repensar los objetos, métodos, teorías y conceptos de la archivología. En consecuencia, podemos considerar que la presencia cada vez mayor de los archivos sonoros como ámbito de reflexión académica, social, política y cultural coincide con la incorporación de las tecnologías de información y comunicación. 


\section{Origen y evolución de \\ los archivos sonoros}

Hace más de 150 años la invención del fonoautógrafo determinó que fuera posible fijar el sonido, gracias a que en 1860 el científico francés ÉdouardLéon Scott grabó la canción Au clare de la lune (Giovannoni, 2008). No fue hasta 1877 que Thomas Alva Edison inventó el fonógrafo (Hill, 2012) y con ello fue posible grabar y reproducir el sonido. Estos dos hechos determinaron el momento histórico en que fue posible fijar el sonido e inició la creación de los primeros documentos sonoros; es decir, la documentación de la historia de la humanidad mediante grabaciones sonoras.

La posibilidad de fijar el sonido se erigió como la nueva tecnología de grabación sonora que captó el interés de la sociedad y motivó la exploración de las posibilidades que ésta tenía en ámbitos como la investigación científica, las artes y la industria del entretenimiento. Sarmiento (2010) recupera uno de los primeros artículos en relación con el fonógrafo que fue escrito por Edison en 1878. En este documento se describen los usos futuros del fonógrafo, entre los que señala los fines educativos, el registro de música, la creación de un archivo familiar, el libro fonográfico, la caja de música, la publicidad y los discursos, entre otros.

Schüller (2008) ha señalado que a principios del siglo XX la investigación científica en disciplinas como la dialectología, la etnolingüística, la etnomusicología y la antropología se apoyó en el uso de la tecnología de grabación sonora, lo que motivó la creación de las primeras fonotecas. El primer archivo sonoro del mundo fue la Phonogrammarchiv de la Academia de Ciencias y Artes de Viena (Phonogrammarchiv der Akademie der Wissenschaffe), creada en 1899 (Miranda Regojo, 1990; Schüller, 2008). Un año después, en 1900, se creó la Phonogrammarchiv de Berlín; en 1908 fue fundada la Phonogrammarchiv de San Petesburgo y la de Zürich en 1909 (Schüller, 2008).

La invención de las primeras grabadoras portátiles de alambre con batería favoreció las grabaciones de investigaciones de campo y posibilitó el registro del lenguaje, la música y los rituales en todo el mundo. En consecuencia, una parte del conocimiento actual de la diversidad lingüística y cultural se basa en los documentos sonoros que se han acumulado en los últimos 50 años (Schüller, 2008). Desde la perspectiva de las artes, Sarmiento (2010) establece que con la irrupción de los movimientos de vanguardia de principios del siglo XX, el fonógrafo se transformó en un instrumento de difusión del arte y del pensamiento y, sobre todo, en un soporte de creación.

El acopio sistemático de documentos sonoros se llevó a cabo de 1920 a 1930. En este periodo surgieron los primeros archivos sonoros de alcance na- 
cional, destinados a salvaguardar la producción fonográfica de inicios del siglo XX; un ejemplo de ello fue la Discoteca dil Stato d'Italia creada en 1928.

En la década de los 30 se comenzaron a grabar los programas radiofónicos (Rooks, 2010). Hay evidencia de que en los primeros años de la radio, una gran cantidad de los programas producidos se perdieron irremediablemente, dado que durante mucho tiempo los miles de programas no tuvieron otro valor que el uso inmediato de la transmisión radiofónica (Rodríguez, 2012).

En 1932 se comenzó a utilizar el vocablo fonoteca, introducido por Gabriel Timmory, para denominar a la Fonoteca Nacional Francesa (Bellveser, 1999; Miranda Regojo, 1990). A finales de los años treinta se integraron las nacientes colecciones sonoras a las bibliotecas (Schüller, 2012). De ello da cuenta la incorporación, en 1938, de la Fonoteca Nacional de Francia a la Biblioteca Nacional.

La consolidación de los archivos sonoros y audiovisuales ocurrió a partir de 1940, debido en gran medida al florecimiento de la industria fonográfica, de manera específica con la introducción del disco de vinilo (Schüller, 2012). Klijn y Lusenet (2008) han señalado que a mediados del siglo XX, en las primeras décadas después de la Segunda Guerra Mundial, fueron creadas más instituciones nacionales de audio y film.

A partir de 1956 las radiodifusoras comenzaron a utilizar la cinta magnética de audio; en 1957 Radio Francia presentó, durante la Exposición Internacional de Bruselas, grabaciones de voces de escritores como material que complementaba la exposición visual (Miranda, 1990).

La investigación científica, la industria discográfica, la radio y la experimentación artística sonora constituyen los sectores creativos de la producción sonora que contribuyeron a la generación de los primeros archivos sonoros. De éstos, el mayor productor de documentos sonoros fue durante varias décadas la radio (Rodríguez, 2012).

\section{Principios de la preservación sonora analógica}

Los programas de radio, la diversidad lingüística de los pueblos originarios, las voces de personajes que han labrado nuestra historia, el testimonio de artistas, científicos, escritores, deportistas y políticos, los géneros musicales y el paisaje sonoro, entre otros, han sido grabados y resguardados en archivos sonoros (también denominados como fonotecas), los cuales se erigieron como las nuevas instituciones de la memoria y basaron sus fundamentos conceptuales, filosofía y principios en instituciones como las bibliotecas, archivos y 
museos, que desde hace siglos son reservorios del patrimonio de la humanidad (Edmondson, 2004).

Las grabaciones sonoras que se resguardan en un archivo sonoro documentan o consignan algo con un propósito intelectual deliberado (Unesco, 2002) y están formadas por el contenido informativo y el soporte en el que se grabó la información. El contenido de un documento sonoro se ha grabado en soportes que han sido creados con diversos tipos de materiales: cera, vinil, baquelita, acetato, papel y plástico, entre otros. Cada soporte de grabación sonora corresponde a una tecnología de grabación y reproducción sonora. Entre otras tecnologías podemos citar el fonógrafo, el gramófono, la grabadora de hilo magnético, el tocadiscos, la grabadora y reproductora de cintas de carrete abierto, así como el disco compacto. El contenido y el soporte habían sido dos componentes estrechamente relacionados e igual de importantes en los soportes analógicos de surcos de sonido (cilindros, los discos de surco grueso, transcripción, lacado y microsurco, etc.); en los soportes analógicos magnéticos (alambre, cinta magnética de carrete abierto, casete de cartucho y cartucho) y en los primeros soportes digitales de sonido (disco compacto, DAT, DVD y Blu-ray) (Edmondson, 2004; Rodríguez, 2012).

Se sabe que la permanencia de los soportes y en consecuencia la información sonora grabada no puede extenderse indefinidamente, por esta razón se debe hacer el mejor esfuerzo para conservarlos en condiciones que permitan su acceso y su uso el mayor tiempo posible (IASA, 2005). Los primeros procesos documentales que se aplicaron en los archivos sonoros derivaron de la experiencia de las bibliotecas y con el paso del tiempo se fueron desarrollando procesos específicos, de acuerdo con la naturaleza sonora de este tipo de documentos.

Dado que la tarea principal de todo archivo sonoro es preservar los documentos sonoros, se debe advertir que términos como conservación, preservación y restauración se utilizan de manera confusa, indistinta y en ocasiones como sinónimos. Conviene señalar la diferenciación que ofrece Voutssas Márquez (2009: 9) en relación con las definiciones de preservación, conservación y restauración: preservar es "cuidar la permanencia de los documentos a largo plazo. Para ello, debemos conservar, esto es protegerlos y resguardarlos anticipada, adecuada y permanentemente; en caso de deterioro o de daño los debemos restaurar." Para efectos de este artículo se retomará la definición que Edmondson (2004: 20) ofrece en relación con la preservación, entendida desde la perspectiva de los archivos sonoros y audiovisuales como "la totalidad de las medidas necesarias para asegurar el acceso permanente-por siempre-de un documento sonoro audiovisual con el máximo de integridad". Las principales asociaciones internacionales de archivos sonoros y audiovisuales, 
como son la Federación Internacional de Archivos de Televisión (FIAT-IFTA), Asociación Internacional de Archivos Sonoros y Audiovisuales (IASA), la Asociación de Archivistas de Imágenes en Movimiento (AMIA), la Organización de las Naciones Unidas para la Educación, la Ciencia y la Cultura (Unesco) y el Consejo Coordinador de las Asociaciones de Archivos Audiovisuales (CCAAA) están de acuerdo con esta definición (Wright, 2012).

La preservación de documentos sonoros analógicos está asociada con la permanencia del soporte físico y establece que el tiempo de vida de todos los soportes de audio está determinado por su estabilidad física y química y por minimizar su uso en el acceso público; es decir, se destaca a la conservación como la medida más importante de la preservación. En relación con esta idea, la IASA (2005) señala que conservar implica almacenar los soportes en ambientes adecuados para este propósito, siempre que sea posible y necesario hay que separar la información primaria de la secundaria y realizar mantenimiento rutinario de limpieza adecuada. La conservación significa tener copias disponibles para acceso y consulta con el fin de minimizar el uso de originales o copias archivo, por lo que una tarea de todo archivo había sido, durante años, la generación de copias para el acceso y consulta como una medida de conservación de los originales del archivo.

De forma complementaria a esta visión, Edmondson (2004) ha establecido que la conservación es el conjunto de elementos necesarios para garantizar la accesibilidad permanente (indefinida) de un documento audiovisual en el máximo estado de integridad. Agrega que sin la catalogación no se pueden identificar ni consultar los documentos sonoros.

Se sabe que la catalogación de documentos sonoros es una tarea especializada que tuvo sus fundamentos en la catalogación de libros, no obstante, la catalogación de documentos sonoros es diferente a la catalogación de libros. Algunas características que diferencian a los documentos impresos de los sonoros son la multiplicidad de tipos de soportes, la diversidad de contenidos y la novedad de la información, la profundidad del análisis de contenido y la ambigüedad del documento (RTVE, 1991). Podemos añadir a estas características la utilización de equipos de reproducción sonora.

Los antecedentes de la catalogación de documentos sonoros datan de 1942, cuando la Music Library Association publicó el Code for Cataloguin Phonograph Records. En 1995 se crearon las Reglas de Catalogación de la Asociación Internacional de Archivos Sonoros y Audiovisuales (IASA), como resultado del trabajo de equipo de profesionales de archivos sonoros y audiovisuales de todo el mundo. Esto significa que durante más de cinco décadas se reflexionó y se analizaron las características y la naturaleza del documento sonoro para crear reglas de catalogación de acuerdo con las características de 
este tipo de documentos. La catalogación de documentos sonoros es el proceso clave para recuperar contenidos y dar acceso a ellos.

Sin acceso, un archivo no tiene sentido ni razón de ser. La conservación y el acceso son dos caras de la misma moneda (Edmondson, 2004). El acceso puede considerarse parte integrante de la conservación. El acceso es definido como todos los usos, exhibiciones o entregas físicas del archivo; es decir, el acceso también es todo uso (Edmondson, 2004) que se haga de los contenidos del archivo, sea media o metadata, a través de servicios de información, publicaciones impresas, multimedia o sonoras u otro tipo de producción. En esta última acepción de acceso se pueden incluir todas las actividades de difusión y reaprovechamiento educativo, cultural o bien comercial, de un archivo sonoro. En suma, el acceso puede ser entendido como el derecho que tiene toda persona a consultar y conocer su patrimonio sonoro.

Conviene señalar que durante muchos años el acceso a los documentos sonoros estuvo condicionado a la disponibilidad de los equipos tecnológicos de reproducción sonora, así como al uso de copias que se ponían a disposición de los usuarios, a través de cabinas o servicios de escucha de discos, casetes y discos compactos, principalmente. Las audiotecas como espacios para la consulta de documentos sonoros eran espacios prácticamente desconocidos.

\section{Incorporación de las tecnologías de información y comunicación en los archivos sonoros}

En 1980 la aparición del disco compacto, como soporte de grabación sonora, coincidió con la formulación de la Recomendación sobre la salvaguardia y la conservación de las imágenes en movimiento emitida por la UNESCO, en la cual se reconoció la importancia cultural, histórica y patrimonial de los documentos audiovisuales, en los cuales se inscriben los sonoros. En ese momento iniciaron las primeras reflexiones en relación con la preservación digital de documentos sonoros (Wright, 2012).

Brylawski (2003) afirma que durante años los archivistas sonoros estuvieron hablando del futuro digital, pero en la actualidad ya no hay más discusiones. El futuro digital llegó. Entre 1989 y 1990, los responsables de los archivos sonoros aceptaron que la intención del clásico paradigma de conservar el material original era un esfuerzo en vano debido a la inestabilidad de los soportes, así como a la futura carencia de algunos equipos de reproducción altamente sofisticados (Schüller, 2008). Incluso, se llegó a afirmar de forma contundente que la preservación analógica estaba muerta, o por lo menos, en un callejón sin salida (Brylawski, 2003). 
De todos los tipos de documentos audiovisuales como son los videográficos, filmográficos y fonográficos, los documentos sonoros fueron los primeros en ser transferidos de una plataforma analógica a una digital. La primera experiencia se llevó a cabo en Alemania en 1992 y estuvo a cargo de la Arbeitsgemeinschaft der öffentlich-rechtlichen Rundfunkanstalten der Bundesrepublik Deutschland/Consorcio de instituciones públicas de radiodifusión de la República Federal de Alemania (ARD) (Haefner, 2001). Siete años más tarde, en 1999, el Institute del Audiovisuel dé Francia (INA) inició su plan de digitalización de archivos (Teruggi, 2004).

Las primeras colecciones en ser digitalizadas fueron las radiofónicas, debido a que con la expansión de los medios masivos de comunicación, como la radio y la televisión, se generó una masa crítica de documentos que debía ser organizada, conservada y archivada (Teruggi, 2004). En el año 2000, en la Conferencia Anual de la Asociación Internacional de Archivos Sonoros y Audiovisuales (IASA), Albrecht Haefner fue uno de los primeros expertos en advertir una nueva tendencia teórica y tecnológica que había comenzado a ganar el reconocimiento de los archivos de la radio y la televisión: la tecnología para el almacenamiento masivo digital (Rodríguez, 2012).

No obstante, conviene destacar que en 1997, cinco años después de que se llevó a cabo la primera digitalizacion, expertos de la Audio Engineering Society (AES), de la National Academy of Recording Arts and Sciences (NARAS) y de la Association for Record Sound Collection (ARSC) coincidieron en señalar que ante la inestabilidad del formato digital se debían guardar los documentos analógicos (CLIRLC, 2006). La recomendación fue considerada como un llamado de mesura ante la inminente presencia de plataformas de preservación digital de archivos sonoros.

Desde esta perspectiva, desde hace más de dos décadas la investigación y la reflexión en relación con el uso de la tecnología para la preservación digital de colecciones sonoras ha sido el motor de las conferencias, foros y seminarios de la Asociación Internacional de Archivos Sonoros y Audiovisuales (IASA) y en la Audio Engeeniering Society (AES), asociaciones internacionales que reúnen a especialistas, investigadores, documentalistas, archivistas, ingenieros y profesionales que durante muchos años han trabajado con archivos sonoros y audiovisuales.

Además de los estudios de fiabilidad de plataformas digitales como medios de preservación de colecciones sonoras, se observó que el costo de los primeros sistemas de almacenamiento masivo digital era muy alto. De ahí que la preservación del patrimonio sonoro en plataformas digitales se tornó un aspecto crítico. Frente a esta realidad, se empezó a reflexionar en torno a la necesidad de contar con opciones que permitieran que la transferencia de 
contenidos analógicos a plataformas digitales estuviera al alcance de un mayor número de archivos sonoros. Esta preocupación fue abordada en la conferenciaBridgingthedigitaldividebyprovidingsuporttocontentprofessionals in the least developer countries, organizada en 2003 por la Unesco. Durante esta conferencia se hizo un llamado para observar que el advenimiento de la brecha tecnológica entre países de primer y tercer mundo también se estaba expandiendo al ámbito de los archivos sonoros y audiovisuales (Unesco, 2003). Un año después, en 2004, en el marco del Quinto Programa en Tecnologías para la Sociedad de la Información la Comisión Europea creó el proyecto PRESTO con el propósito de buscar soluciones al problema de la preservación digital. Este proyecto se centró en buscar nuevas oportunidades de almacenamiento y acceso a largo plazo, 20 años o más (Wright, 2004). La institución líder de este proyecto fue la British Broadcasting Corporation (BBC) y participaron como socios el Institut National del Audiovisuel de Francia (INA) y la Radio Televisione Italiana (RAI).

Además de los estudios e investigaciones desarrollados en el proyecto PRESTO, una de las más significativas aportaciones en el desarrollo de tecnologías para la preservación digital fue formulada por el Kevin Bradley, quien publicóHaciaunsistemadealmacenamientoypreservaciónencódigoabierto. Recomendacionesrespectoalaimplementacióndeunsistemadepreservación de archivos digitales y temas en torno al desarrollo del software, una propuesta para la creación de sistemas de almacenamiento y preservación en código abierto, con la idea de que estuvieran al alcance de las pequeñas instituciones que no cuentan con recursos financieros y que tienen ante síla impostergable tarea de preservar sus colecciones sonoras.

La metodología del proyecto pretendió describir (algo similar a construir) un sistema de almacenamiento masivo digital autónomo en pequeña escala y de depósito al usar software de código abierto. El sistema fue ideado para asumir todas las funciones de archivo normales de un sistema de almacenamiento digital, esto es, administración de tareas e ingesta, administración y extracción de metadatos, preservación y almacenamiento de respaldo (Bradley, 2007b).

El punto de partida de Bradley fue reconocer que debido a que no existen sistemas de almacenamiento digital permanentes, es necesario considerar la creación de un sistema simple y sustentable que provea las estrategias para administrar todas las funciones identificadas para la preservación digital, que se apoye en un código abierto y que desarrolle un sistema de archivos (Bradley, 2007b). En la actualidad, el uso de código abierto constituye una alternativa en la preservación digital de archivos sonoros. 


\section{Digitalización y preservación digital}

Lapreservación digitalyla digitalización son dos conceptosestrechamenterelacionados. Voutssas Márquez (2009) define a la preservación digital como las acciones específicas cuyo fin ulterior y a largo plazo es el de asegurar la permanencia y acceso del contenido de documentos digitales a lo largo del tiempo y las tecnologías, de manera independiente de su soporte, formato o sistema. Para lograrlo es necesario mantenerlos, lo que equivale a protegerlos y resguardarlos anticipada y permanentemente; en caso de deterioro o daño debemos tratar de restaurarlos.

Desde la perspectiva de Térmens (2013), la preservación digital asegura el acceso y el uso futuro de los documentos digitales creados en el presente o en el pasado, a partir de las políticas de conservación y de seguridad informática que aseguran su mantenimiento y uso a largo plazo. Romero (2006) considera que la preservación digital se desarrolla como una tarea global, continua y compleja en la que han de tenerse en cuenta factores físicos y lógicos de la información, así como un tratamiento de descripción formal correcto $y$ adecuado.

En consecuencia, la preservación digital de archivos sonoros es el método sustentable mediante el cual se conservan, administran, gestionan y se proporciona acceso, difusión y reaprovechamiento permanente del audio digital, también denominado esencia o media y los metadatos.

Por su parte, la digitalización es la transferencia de contenidos en soportes analógicos a digitales; es decir, el proceso por el cual la señal analógica es reemplazada por una señal digital (IASA, 2005). La digitalización permite proteger valiosos documentos de la manipulación y el deterioro. Es la única manera de asegurar la supervivencia del material audiovisual (Unesco-UBC, 2012). Más allá de ser el proceso de transferencia y conversión de contenidos analógicos en plataformas digitales, es una estrategia de gestión de archivos (Green, 2006).

A través del proceso de digitalización se convierte un determinado contenido, por ejemplo, el audio de una grabación, en una serie de valores numéricos. De acuerdo con las recomendaciones de expertos e investigadores (IASA, 2005; ARSC-Technical Committee, 2011) la digitalización se basa en las siguientes recomendaciones:

1. La digitalización se debe realizar sin compresión de datos, con base en los parámetros consensuados de muestreo y cuantificación de la señal digital. 
2. Se debe realizar la recuperación óptima de la señal analógica del documento sonoro con los equipos adecuados de grabación y reproducción sonora.

3. La digitalización se ha de realizar sin alterar el documento original.

4. Se debe incorporar un vínculo entre la media y la metadata a través de la clave alfanumérica de identificación (ID).

La digitalización es una medida para la preservación de documentos grabados en soportes analógicos y el punto de partida para la aplicación de métodos de preservación digital.

Una vez definidos los términos digitalización y preservación digital, conviene establecer que cada día se producen miles de documentos sonoros cuyo origen es digital. De ahí que la preservación digital de documentos sonoros sea un tema complejo, que debe considerar tanto la salvaguarda de los contenidos derivados de la digitalización como de aquéllos cuyo origen es digital.

La preservación digital incluye acciones de intervención y de conservación. La migración (desde un formato de archivo obsoleto a un formato actual) constituye otra tarea de preservación. La prevención o el retraso de deterioro y daño son acciones de conservación que no evitan la obsolescencia (Wright, 2012). De acuerdo con la definición ofrecida por Wright, la migración sería parte de las medidas de preservación, y la conservación de los archivos digitales es la tarea permanente en la preservación digital.

\section{Efecto de la preservación digital en los archivos sonoros}

Hace más de una década Chen (2001) advirtió que los conceptos de conservación y preservación se transformarían con la presencia de la preservación digital. Este cambio ha sido tan significativo que ha modificado la salvaguarda de los archivos sonoros a través del advenimiento de nuevos flujos y perfiles profesionales, la generación de copias digitales sin pérdida, la incorporación de la media y la metadata como componentes básicos de la preservación digital, la creación de los sistemas de gestión y almacenamiento masivo digital y la extensión del acceso, difusión y reaprovechamiento de los documentos sonoros. Además, este cambio confronta una serie de desafíos derivados de la preservación digital de documentos sonoros.

\section{Nuevos flujos y perfiles profesionales}

La digitalización fue el nuevo primer proceso que se incorporó en las tareas de un archivo sonoro tradicional. Desde la perspectiva de Westerhof (2011) 
la digitalización es la raíz de los cambios de organización y la generación de problemas en los archivos, este proceso también tiene efecto en la manera de pensar las colecciones del archivo. A partir de la digitalización, de forma progresiva se han incorporado nuevos flujos de trabajo en un archivo como la identificación e ingreso del documento digital a través de la digitalización o ingesta, la validación y la verificación, tanto de la catalogación como de la digitalización; acceso in situ y remoto al documento sonoro, verificación permanente de la integridad y consistencia tanto del audio digital como de la metadata y la realización de copias de seguridad de la media y la metadata de forma periódica, en función de las políticas establecidas por el archivo. Por tanto, se requiere que los profesionales que se hagan cargo de estas tareas cuenten con nuevas competencias profesionales. En la experiencia de digitalización de los Archivos Nacionales de Imagen y Sonido de Holanda, uno de los problemas principales fue el relativo al personal. La digitalización generó cambios en diferentes niveles. El primero y principal cambio fue en el equipo de trabajo. Las competencias cambiaron. La incorporación de nuevo personal trajo puntos de vista y herramientas dramáticamente diferentes a las utilizadas por los archivistas tradicionales. Diferentes culturas vinieron a encontrarse y esto no siempre es fácil de comprender por parte de los archivistas (Westerhof, 2011).

Los archivos han de hacer frente a la doble incógnita de la preservación digital, por un lado, la continuidad de la conservación de los formatos tradicionales anteriores y, por otro, la demanda de acceso a éstos (Edmondson, 2004). Es decir, aunque se empleen plataformas para la preservación digital de colecciones sonoras, deberán seguir desarrollándose procesos documentales tradicionales como la conservación de fonorregistros en bóvedas, lo que conlleva considerar la coexistencia de métodos de preservación de colecciones analógicas y colecciones digitales.

\section{Copias digitales sin pérdida}

Hemos dicho que conservar el contenido (no los soportes) significó un cambio clave de la preservación digital. Durante muchos años la realización de copias en soportes analógicos fue un trabajo que se realizó con una pérdida constante en la calidad del documento. Con la digitalización ya no se hacen copias en soportes analógicos, ahora la única forma que existe para tener copias y garantizar la preservación a largo plazo de los archivos es transferirlos a una plataforma digital.

En el documento digital el contenido se separa del soporte. Esta separación es el principio de la nueva copia de preservación, es decir, una clonación 
del documento original que es completamente idéntico (Wright, 2012). Se incorpora como nuevo concepto la copia de mayor fidelidad (loss less copiyng), lo que significa que la copia es idéntica al original y esto sólo es posible en el dominio digital (Schüller, 2006, 2012). En consecuencia, la prioridad para la preservación digital es la conservación de los contenidos digitales.

\section{La media y metadata, componentes básicos}

Como se ha explicado con anterioridad los dos componentes básicos a considerar en la preservación digital son el audio digital, también denominado esencia o media y los metadatos. Para la preservación del audio es indispensable que los formatos, resoluciones, soportes y sistemas tecnológicos se adhieran a los estándares aceptados a nivel internacional. Las versiones fuera de los estándares no representan caminos seguros para una migración e intercambio de información a largo plazo y de formatos a futuro (IASA, 2006).

Los metadatos constituyen la información fundamental para el uso y administración de las colecciones sonoras una vez digitalizadas. Los metadatos no son sólo un medio para identificar y estructurar información, sino para hacerla recuperable (De Jong, 2001). Los metadatos de un archivo sonoro digital están formados por la información que proviene de la catalogación, la digitalización y la administración del documento sonoro (De Jong, 2001). Un archivo digital bien planeado deberá automatizar la producción de sus metadatos e incluir información acerca del soporte original de grabación, el formato y estado de conservación, el equipo y los parámetros de reproducción, la resolución digital del formato, todo el equipo empleado, los operadores participantes y cualquier otro proceso o procedimiento que se haya involucrado (IASA, 2006).

Los metadatos en un archivo sonoro son las herramientas clave para la comunicación entre los sistemas actuales y los nuevos que surjan en el campo tecnológico. Sin metadatos no es posible el intercambio de información digital, cualquiera que ésta sea (De Jong, 2001). Un archivo sonoro requiere documentar todos los cambios que se hagan en la estructura de los metadatos. Los registros de metadatos, si se crearon y se mantuvieron correctamente, podrán asegurarse contra cualquier cambio relevante o de almacenamiento que se aplique en el campo apropiado (Rodríguez, 2012).

\section{Sistema de Gestión y Almacenamiento Masivo Digital}

A lo largo de los años, los archivos sonoros y audiovisuales han tenido como reto principal negociar el espacio de almacenamiento que conforme pasaba 
el tiempo se incrementaba (Wright, 2011). La preservación digital modificó la concepción del almacenamiento masivo en bóvedas ante la aparición del Sistema de Gestión y Almacenamiento Masivo Digital (SGAMD).

El Comité Técnico de la IASA (2006: 54) define al Sistema de Gestión y Almacenamiento Masivo Digital como "Un sistema comprensible, completamente automatizado y diseñado para almacenar, administrar, mantener, distribuir y preservar un complejo conjunto de objetos digitales heredados junto con los metadatos relacionados, un sistema de respaldo y almacenamiento sencillo".

Los sistemas de gestión y almacenamiento masivo digital integran y automatizan los procesos para controlar, digitalizar, almacenar, catalogar, administrar y distribuir objetos digitales y metadatos de un archivo sonoro con el propósito de garantizar la preservación y el acceso. Los SGAMD establecen los fundamentos para la operación de un archivo digital que pueda adoptar modelos de archivo abierto como el OAIS (Open Archival Information System) y de administración de metadatos como el PREMIS. Los SGAMD son las plataformas tecnológicas para la preservación digital de un archivo sonoro.

\section{Acceso, difusión y reaprovechamiento del archivo sonoro}

El acceso, visibilidad y reaprovechamiento del archivo sonoro es uno de los cambios más relevantes derivados de la preservación digital. Thibodeau (2010) ha señalado que el valor de la preservación digital se realiza en el uso de la información preservada. El fin último de la conservación, por tanto, debe ser optimar las posibilidades de uso.

La posibilidad de que un mayor número de personas puedan consultar el archivo amplía geográficamente las posibilidades de acceso de un archivo sonoro a través de Internet, además, posibilita la interacción con los usuarios en las redes sociales. Se crean nuevos servicios especializados derivados de los catálogos sonoros de un archivo, lo que lleva a la puesta en valor de los documentos de cada archivo. Asimismo, la preservación digital constituye un cambio fundamental en el fomento de una cultura de la escucha. Desde esta perspectiva, actividades como la selección de una fonoteca básica para niños de educación preescolar, la creación de un mapa sonoro o el desarrollo de mapas de contenidos educativosy culturales constituyen sólo una muestra de la amplia variedad de opciones que el reaprovechamiento de un archivo sonoro tiene ante sí. Un archivo sonoro adquiere vitalidad y hace de los fonorregistros resguardados un patrimonio vivo y un componente fundamental de la sociedad contemporánea. 


\section{Desafíos de la preservación digital}

Los desafíos han de ser entendidos como los retos que la preservación digital de archivos sonoros conlleva para garantizar la preservación digital a largo plazo. De todos los desafíos conviene señalar los siguientes.

\section{Obsolescencia y migración}

Thibodeau (2010) ha establecido que la única predicción válida para la tecnología de la información es el cambio constante. En consecuencia, la preservación digital concebida como una solución permanente para la salvaguarda del archivo sonoro, que resuelve los problemas de fragilidad y obsolescencia de los soportes de grabación producidos hace más de un siglo y medio, ya no será funcional en un periodo relativamente corto. Como la tecnología madura y la densidad de almacenamiento se incrementa, con frecuencia el hardwarecomienza a ser obsoleto, mientras que los procesadores de computadorasy muchas aplicaciones de software dejan deemplearsey hacen que los recursos no puedan ser consultados; por tanto, cualquier decisión sobre la preservación digital debe incorporar la capacidad de cambio tecnológico. Al respecto, Bradley (2007a) considera necesario tener una visión sustentable y a largo plazo en la preservación digital de un archivo sonoro.

En consecuencia, la migración será una tarea permanente. Schüller (2006) y Teruggi (2004) coinciden en señalar que la migración de información hacia nuevos sistemas de almacenamiento, una vez que los sistemas sean técnicamente obsoletos, es uno de los aspectos claves para la preservación digital. La migración es entonces un proceso cíclico que, de acuerdo con SchüIler (2006), no deber ser mayor a diez años.

\section{Fallas de la tecnología y errores humanos}

La preservación digital también es frágil debido a los riesgos inherentes del uso de la tecnología y a los errores humanos. Van Malssen (2011) ha establecido que algunos de los riesgos comunes a los medios digitales, que pueden ser aplicados a la preservación digital de archivos sonoros, son los errores de operación de tecnología (software y hardware), los derivados de la transferencia de archivos y los ataques internos y externos.

Por otra parte, los errores humanos de operación del sistema de gestión y almacenamiento masivo digital provienen de la falta de formación y capacitación especializada en el manejo de este tipo de tecnologías (Van Malssen, 2011). Debido a que la preservación es un nuevo ámbito de conocimiento, en muchas 
instituciones no se cuenta con el personal calificado para el manejo de la tecnología. En ocasiones, se incorpora a personal técnico sin los conocimientos suficientes y esto genera errores humanos que afectan la operación del archivo digital. Más aún, la capacidad de respuesta para enfrentar un ataque informático interno o externo se relaciona directamente con la capacidad de contar con personal calificado que pueda hacer frente a estas situaciones imponderables.

\section{Continuidad social, económica y politica}

A menudo, la continuidad social, económica y política es quizás la más grande amenaza. Si la institución decide no apoyar el mantenimiento de la preservación digital, los recursos digitales pueden desaparecer. Incluso en la Declaración de Vancouver (Unesco-UBC, 2012: 1) uno de los primeros pronunciamientosafirmabaque"sepierdenconstantementegrandescantidades de información debido a que se desconoce su importancia, no existen marcos legales e institucionales para garantizar su conservación y hace falta una mejor capacitación y financiación".

En este sentido Wright (2012: 15) señaló:

En el mundo físico (analógico) es fundamental el medio ambiente, control de temperatura y humedad relativa, el sistema de prevención de incendios y adecuadas instalaciones de almacenamiento. En el mundo de la preservación digital los temas a considerar son los entornos sociales, políticos y económicos, así como la gestión de un archivo que no se ve.

La continuidad en la preservación digital de un archivo sonoro está asociada a la sustentabilidad a largo plazo.

\section{Desastres naturales}

Los desastres naturales como las inundaciones, terremotos e incendios, entre otros, ponen en riesgo tanto colecciones analógicas como digitales, por lo que una de las principales consideraciones que garantiza la seguridad de un archivo sonoro en plataformas digitales es contar cuando menos con dos copias ubicadas en diferentes lugares, así como con un plan de recuperación en caso de desastre (Van Malssen, 2011; Unesco-UBC, 2012).

\section{Falta de metadata}

La falta de metadata representa una amenaza en el manejo de colecciones grandes de documentos digitales (Van Malssen, 2011). La carencia de me- 
tadatos que permitan identificar los contenidos de una colección sonora es grave en archivos analógicos, pero es más grave en colecciones digitales en las cuales no se puede ver el soporte. Antes de iniciar la digitalización de las colecciones sonoras se debe contar con un inventario, como mínimo, a fin de disponer de la metadata básica que permita la identificación del documento digital.

\section{Conclusiones}

Hace más de un siglo y medio que fue posible fijar el sonido. A partir de entonces se han producido millones de documentos sonoros que dan cuenta de la historia y la creación artística, cultural, científica y política contemporánea expresada en sonidos; por tanto, el documento sonoro forma parte del patrimonio de la humanidad. En consecuencia, la preservación de colecciones analógicas fue durante mucho tiempo la principal encomienda de archivos, bibliotecas, fonotecas e instituciones de resguardo de la memoria sonora.

La raíz de un cambio fundamental en los archivos sonoros se generó al reconocer que conservar el material original era un esfuerzo en vano debido a la inestabilidad de los soportes, así como a la futura carencia de equipos de reproducción.

Con el advenimiento de la preservación digital la prioridad fue preservar el contenido y no el soporte; en consecuencia, los archivos sonoros experimentan una transformación que puede observarse a través de la incorporación de nuevos flujos de trabajo y perfiles profesionales definidos por la coexistencia de métodos de preservación analógica y digital, la realización de copias digitales sin pérdida que ha modificado la idea de documento original, la incorporación de la media y la metadata como los componentes básicos de un archivo digital, el uso de Sistemas de Gestión y Almacenamiento Masivo Digital, así como la posibilidad de un mayor acceso, difusión y reaprovechamiento del documento sonoro.

La preservación digital produce en los archivos sonoros un cambio que implica considerar una serie de desafíos, entre los que se encuentran la obsolescencia de la tecnología y migración como tarea permanente, las fallas de la tecnología y los errores humanos, la continuidad social, económica y política, los desastres naturales y la falta de metadata. 


\section{Referencias}

ARSC-Technical Committee (2011), Study of Embedded Metadata Support in Audio Recording Software. Association of Recorded Sound Collections. Disponible en www.arsc-audio.org/pdf/ARSC_TC_ MD_Study.pdf [Fecha de consulta: 30 de marzo de 2014].

Ávila, Araujo (2013), "Integração entre Arquivologia, Biblioteconomia e Museologia como marco identitário para a Ciência da Informação", en Miguel Ángel Rendón Rojas, El objeto de estudio de la bibliotecología/Documentación/Ciencias de la Información. Propuestas, discusión análisis y elementos comunes, México: IIBI.

Bellveser, Enric (1999), Manual de documentació audiovisula de radiò i televisió, España: Universidad de Valencia.

Bradley, Kevin (2007a), "Defining digital sustainability", en Library Trends, 56 (1): 148-163.

- (2007b), Hacia un sistema de almacenamiento y preservación en código abierto, trad. al español de Antonio Limas, México: Subcomité de Técnología del Programa Memoria del Mundo de la Unesco, Conaculta

Brylawski, Sam (2003), "Review of Audio Collection Preservation Trends and Challenges", en Memorias del Symposium Sound Saving, Universidad de Texas. Disponible en http://www.arl.org/sto rage/documents/publications/sound-savings.pdf [Fecha de consulta: 30 de marzo de 2014].

y Bambeger, Rob (2010), The State of Recorded Sound Preservation in the United States: A national Legacy at Risk in the Digital Age, Washington, D.C.: Council on Library and Information Resources at the Library Congress.

Castells, M. (2004), La era de la información: economía, sociedad y cultura, vol. 3, México: Siglo XXI Editores.

Chen, S. S. (2001), "The paradox of digital preservation", en Computer, 34 (3): 24-28.

CLIRLC (Council on Library and Information Resources and the Library of Congress) (2006), Capturing Analog Sound for Digital Preservation: Report of a Roundtable Discussion of Best Practices for Transferring Analog Discs and Tapes, Washington, D.C.

Comité Técnico de la IASA (2006), Lineamientos para la producción y preservación deobjetos deaudio digitales, Kevin Bradley(ed.),Suecia: IASA.

Dayli. Jay E. (1967), "The Selection, Processing, and Storage of NonPrint Materials: A Critique of the Anglo-American Cataloging Rules as The Relate to Newer Media", en Library Trends, 16 (288): 283-289.

De Jong, Annemieke (2001), Los metadatos en el entorno de la producción audiovisual, México: Radio Educación.

- (ed.) (2011), Zorgen voor onzichtbare assets. Over het behoud van digitale AV-collecties, Hilversum: Beeld en Geluid. 
Edmondson, R. (2004), Audiovisual archiving: Philosophy and principles, París: Unesco.

(2010), "Philosophical fundaments in the digital age", conferencia presentada en el Cuarto Seminario Internacional de Archivos Sonoros y Audiovisuales, México.

Giovannoni, David (2008), Principes de phonoautograqaphie (1857). Édouard Léon Scott de Martinville, ed. fasc., Académie des sciences del'Institut de France no. 1639. Disponible en http://www.first sounds.org/ [Fecha de consulta: febrero de 2014].

Green, Richard (2006), "Memoria y preservación digital", en Perla Rodríguez (comp.), Memorias del Tercer Seminario Internacional. La Preservación de la memoria audiovisual en la sociedad digital, México: Radio Educación.

Haefner, Albrecht (2001), "Strategies and preservation polices in the radio archive of the German Public broadcaster SWR", en Perla Rodríguez, Los archivos sonoros y audiovisuales en América Latina. Memorias del Primer Seminario Internacional, México: Radio Educación-Conaculta, 97-112.

Hill, V. Elizabeth (2012), "The preservation of sound recordings", en Music Reference Services Quarterly, 15: 88-98.

IASA (Asociación Internacional de Archivos Sonoros y Audiovisuales) (2005), Normas, prácticas recomendadas y estrategias. IASA TC03. Lasalvaguardiadel patrimoniosonoro:ética,principiosyestrategias de preservación, México: IASA. (2006),Lineamientosparalaproducciónypreservacióndeobjetos de audio digitales TC-04, Unesco.

- (2014). Disponible en: www.iasaweb.org [Fecha de consulta: 30 de marzo de 2014].

Instituto Centrali per i Bieni Sonoro e Audiovisivi. Disponible en http:// www.icbsa.it/index.php?it/129/discoteca-di-stato el 3 de marzo de 2014.

Klijn, E. y Lusenet, Y. D. (2008), Tracking the reel world, Amsterdam: European Commission on Preservation and Access.

Miranda Regojo, Fátima (1990), La fonoteca, Madrid: Fundación Germán Sánchez Ruipérez.

Proyecto PRESTO. Disponible en www.prestocentre.org (enero de 2014).

Rodríguez, Perla (2012), El archivo sonoro. Fundamentos para la creación de una fonoteca nacional, México: Library Outsourcing.

Romero, Jorge Candás (2006), “El papel de los metadatos en la preservación digital", en El profesional de la información, 15 (2): 126-136.

Rooks, S. (2010), "What happen to the BBC sound archive?", en Journal of the Society of Archivist, 31: 177-185.

Rousseau, Jean Yves y Couture, Carol (1994), Les fondements de la discipline arcuivistique, Québec: Presses de l'Université du Québec. 
RTVE (Radio Televisión Española) (1991), Normas de catalogación del Archivo Sonoro de Radio Nacional deEspaña 1.Palabrayefectossonoros, España: Centro de Formación de RTVE.

Sarmiento, José Antonio (2010), La música del vinilo, España: Centro de creación experimental, Taller de ediciones, Universidad CastiIla La Mancha.

Schüller, Dietrich (1997), "Strategies for the safeguarding of audio and video materials in the long term", en Helen Harrison (comp.), Audiovisual Archives a practical reader, París: United Nations Educational Scientific and Cultural Organization Publications -CII WS, 292-297.

(2008), Audiovisual research collections and their preservation, Amsterdam: European Commission on Preservation and Access. Disponible en: http://www.emc.com/leadership/programs/digitaluniverse.htm [Fecha de consulta: 4 de diciembre de 2013].

(2006), "La preservación de la herencia digital”, en Perla Rodríguez (comp.), Memorias del Tercer Seminario Internacional. La Preservación delamemoriaaudiovisualen la sociedaddigital,México: Radio Educación.

(2012), "The Role of Digitization in the Preservation of Audiovisual Documents", en Preservation of Digital Information in the Information Society. Proceedings of the International Conference, Moscow, 3-5 de octubre de 2011. Disponible en: http://www.ifap com.ru/files/News/Images/2013/dig_pres_is_eng.pdf [Fecha de consulta: 30 de marzo de 2014].

Térmens, Miquel (2013), Preservación digital, Barcelona: Editorial UOC.

Teruggi, D. (2004), “Electroacoustic preservation projects: how to move forward", en Organised Sound 9 (1): 55-62. DOI: 10.1017/51 355771804000081

Thibodeau, Kenneth (2001), "Building the archives of the future: advances in preserving electronic records at the National Archives and Records Administration", en D-Lib Magazine, 7 (2), febrero. (2010), “Digital preservation: communicating across cyberspace and time", en Memorias de 2010 Roadmap for Digital Preservation Interoperability Framework Workshop, ACM.

- (2012), "Wrestling with Shape-Shifters Perspectives on Preserving Memory in the Digital Age", conferencia presentada en The Memory of the World in the Digital age: Digitization and Preservation, 26-28 de septiembre de 2012, Vancouver, British Columbia, Canadá.

Unesco (2002), Memoria del mundo. Directrices para la salvaguardia del patrimonio documental, Ray Edmondson (ed.), París. (2003),Bridging the digitaldivideby providing supporttocontent professionalsintheleastdevelopercountries,conferenciaorganizada por la Comisión Suiza de la UNESCO, Lugano, Suiza. 
Unesco-UBC (2012), Declaración de Vancouver. La memoria del Mundo en la era digital: digitalización y preservación, Vancouver.

Van Malssen, Kara (2011), "Planing beyond digitization: digital preservation of audiovisual collections", en Annemieke De Jong, Zorgen voor onzichtbare assets. Overhet behoud van digitale AV-collecties. Hilversum: Beeld en Geluid, 168-191.

Vieira, Thiago de Oliveira (2013), "O risco da patrimonialização: a (não) avaliação e seleção dos documentos audiovisuais, iconográficos e sonoros", en Ponto de Acesso, Salvador, 7 (3): 148-166.

Vivas Moreno, Agustín (2013), "Concepto de archivística, archivo y gestión de documentos: definición y análisis", en Miguel Ángel RendónRojas, Elobjetodeestudiodelabibliotecología/Documentación/Ciencias de la Información. Propuestas, discusión análisis y elementos comunes, México: UNAM, Instituto de Investigaciones Bibliotecológicas y de la Información.

Voutssas Márquez, J. (2009), Preservación del patrimonio documental digital en México, México: UNAM, Centro Universitario de Investigaciones Bibliotecológicas.

Weihs, J. A. (2001), "Somewhat personal history of non book cataloguing", en Sandra Roe (ed.), The Audiovisual Cataloguing current, 31 (3), New York: The Haworth Information Press, 159-175.

Westerhof, Hans (2011), "Mass digitisation: lessons from a managerial perspective", en Annemieke De Jong, Zorgen voor onzichtbare assets. Over het behoud van digitale AV-collectties, Hilversum: Beeld en Geluid.

Wright, Richard (2012a), Preserving Moving Pictures and Sound, Gran Bretaña: Digital Preservation Coalition. DPC Technology Watch 12-01 March 2012. Disponible en http://dx.doi.org/10.7207/twr12 -01 [Fecha de consulta: febrero de 2014].

(2011), "How can invisible files stored on masstorage perhaps even in the cloud ever claim authenticity?", en Zorgen voor Onzichtbare Assets Over het behoud van digitale AV-collecties, Hilversum: Beeld en Geluid.

(2004), "Digital preservation of audio, video and film", en VINE. The Journal of the Information and Knowledge Management Systems, 34 (2), 71-76.

Para citar este artículo como revista electrónica:

Rodríguez Reséndiz, Perla Olivia. 2016. “La preservación digital sonora".Investigación Bibliotecológica:Archivonomía, Bibliotecologíae Información.68:173-195.Aquíseagregaladirecciónelectrónica(Consultado el día-mes-año) 
Para citar este artículo tomado de un servicio de información:

Rodríguez Reséndiz, Perla Olivia. 2016. “La preservación digital sonora". Investigación Bibliotecológica: Archivonomía, Bibliotecología e Información.68:173-195.En:Aquíseagregaelnombredelserviciodeinformación y la dirección electrónica (Consultado el día-mes-año) 
